\title{
A NOTE ON MT OPERATORS
}

\author{
D. A. TRAUTMAN ${ }^{1}$
}

\begin{abstract}
We give two characterizations of an MT operator $A$ from a Banach space $X$ into a quasi-Banach space $Y$. Both characterizations are generalizations of results of Burkholder concerning MT spaces, and one characterization answers a question of Burgain and Davis.
\end{abstract}

1. Preliminaries. Let $X$ be a vector space over the complex field $\mathbb{C}$ or the real field $\mathbf{R}$. We say that $X$ is a quasi-Banach space if there exists a complete real-valued function $\|\cdot\|$ on $X$ such that for all $x, y \in X$ and all scalars $\alpha$

$$
\|x\|=0 \quad \text { iff } x=0, \quad\|\alpha x\|=|\alpha|\|x\|, \quad\|x+y\| \leq K(\|x\|+\|y\|)
$$

where $K \geq 1$ is independent of $x$ and $y$. The function $\|\cdot\|$ is called a quasi-norm. If $K=1$, we say $X$ is a Banach space and $\|\cdot\|$ is a norm. By the Aoki-Rolewicz theorem [4] we may assume there exists $q, 0<q \leq 1$, such that $\|\cdot\|$ is $q$-subadditive. That is, for $x, y \in X$,

$$
\|x+y\|^{q} \leq\|x\|^{q}+\|y\|^{q} .
$$

Let $X$ be a Banach space, $(\Omega, A, P)$ a nonatomic probability space and $A_{0}, A_{1}, \ldots$ a nondecreasing sequence of sub- $\sigma$-fields of $\mathcal{A}$. A sequence $f=\left(f_{n}\right)$ of $X$-valued functions is a martingale if the corresponding difference sequence $d=\left(d_{n}\right)$ given by $d_{1}=f_{1}$ and $d_{n}=f_{n}-f_{n-1}$ for $n \geq 2$ satisfies

$$
\begin{aligned}
& d_{n}: \Omega \rightarrow X \text { is strongly measurable with respect to } A_{n}, \\
& \left\|d_{n}\right\|_{L_{1}(X)}=E\left(\left\|d_{n}\right\|\right)<\infty, \\
& E\left(d_{n+1} \mid A_{n}\right)=0, \quad n \geq 1,
\end{aligned}
$$

where $E$ denotes expected value.

A sequence $u=\left(u_{n}\right)$ of scalar-valued functions is a predictable sequence if $u_{n}$ is $\mathcal{A}_{n-1}$-measurable. Here we shall always assume also that $\sup _{n}\left\|u_{n}\right\|_{\infty} \leq 1$. The transform $g=\left(g_{n}\right)$ of $f$ by $u$ is given by

$$
g_{n}=\sum_{j=1}^{n} u_{j} d_{j} .
$$

We also use $f^{*}(\omega)=\sup _{n}\left\|f_{n}(\omega)\right\|$ and for $1 \leq p<\infty$

$$
\|f\|_{L_{p}(X)}=\sup _{n}\left\|f_{n}\right\|_{L_{p}(X)} .
$$

Received by the editors January 11, 1985 and, in revised form, April 17, 1985.

1980 Mathematics Subject Classification. Primary 46B20, 46E40, 60G46.

Key words and phrases. Martingale transforms, MT operators, quasi-Banach spaces.

${ }^{1}$ Research supported in part by an Ohio State University Seed Grant. 
If $Y$ is a quasi-Banach space and $A: X \rightarrow Y$ is continuous and linear, then for $1 \leq p<\infty$

$$
\|A g\|_{L_{p}(Y)}=\sup _{n}\left\|A g_{n}\right\|_{L_{p}(Y)}
$$

Following Bourgain and Davis [1], we make the following definition:

Definition. We say a continuous linear operator $A: X \rightarrow Y$ from a Banach space into a quasi-Banach space is an $M T$ (resp. UMD) operator if for each $p$, $1<p<\infty$, there exists a constant $C=C(p)$ so that, for all $X$-valued martingales $f=\left(f_{n}\right)$, all predictable sequences $u=\left(u_{n}\right)$ (resp. all choices of sign $\varepsilon=\left(\varepsilon_{n}\right)$, $\left.\varepsilon_{n}= \pm 1\right)$ and transforms $g$ of $f$ by $u$ (resp. $\varepsilon$ ) we have $\|A g\|_{L_{p}(Y)} \leq C\|f\|_{L_{p}(X)}$.

Burkholder [3] defines a Banach space $X$ to be an MT (UMD) space if the identity operator on $X$ is an MT (UMD) operator. He then shows that $X$ is an MT space if and only if it is an UMD space. Perhaps inspired by this, Bourgain and Davis [1] asked if an UMD operator is an MT operator. In $\S 2$ we answer in the affirmative. Burkholder [3] also gives a geometric characterization of an UMD space. In $\S 3$ we give a geometric characterization of an UMD operator.

I take this time to thank Bill Davis for introducing me to this material, Neal Carothers for some helpful comments, and the referee for some useful suggestions concerning the rewriting of the first draft of this paper.

\section{Equivalence of MT and UMD.}

THEOREM 1. Let $A: X \rightarrow Y$ be a continuous linear operator from a Banach space $X$ to a quasi-Banach space $Y$. Then $A$ is an MT operator if and only if $A$ is an UMD operator.

Proof. Clearly we need only show UMD implies MT. So let $f=\left(f_{n}\right)$ be an $X$-valued martingale with difference sequence $d=\left(d_{n}\right), u=\left(u_{n}\right)$ a predictable sequence and $g$ the transform of $f$ by $u$. We need only show that if $\|f\|_{L_{p}(X)}<\infty$, then for all $n \geq 1$

$$
\left\|A g_{n}\right\|_{L_{p}(Y)} \leq C\|f\|_{L_{p}(X)}
$$

where $p, 1<p<\infty$, is fixed, and $C$ is a constant independent of $f, u$ and $n$. By considering the real and imaginary parts of $u$ separately (in case $C$ is our scalar field) we may assume $u$ is real-valued.

So fix $n$. Following Burkholder's methods in [2 and 3] we may make the following assumptions: We assume that $f_{1}, \ldots, f_{n}$ are simple functions, and we assume that $u_{k}$ is single-valued with $u_{k} \in[-1,1]$ for $1 \leq k \leq n$. Thus write $u_{k}=\sum_{i=1}^{\infty} 2^{-i} \varepsilon_{i k}$, $\varepsilon_{i k}= \pm 1$.

As noted earlier we may assume $\|\cdot\|_{Y}$ is $q$-subadditive for some $0<q \leq 1$. Write $p=q p^{*}$ where $1<p \leq p^{*}<\infty$. Then by the subadditivity of $\|\cdot\|_{Y}^{q}$, the 
subadditivity of the $L_{p^{*}}$ norm and the UMD property of $A$, we have

$$
\begin{aligned}
\left\|\sum_{k=1}^{n} A\left(u_{k} d_{k}\right)\right\|_{L_{p}(Y)}^{q} & =\left\|\sum_{i=1}^{\infty} 2^{-i} \sum_{k=1}^{n} A\left(\varepsilon_{i k} d_{k}\right)\right\|_{L_{p}(Y)}^{q} \\
& =\left(\int_{\Omega}\left\|\sum_{i=1}^{\infty} 2^{-i}\left(\sum_{k=1}^{n} A\left(\varepsilon_{i k} d_{k}\right)\right)\right\|_{Y}^{p} d P(\omega)\right)^{1 / p^{*}} \\
& =\left(\int_{\Omega}\left(\left\|\sum_{i=1}^{\infty} 2^{-i}\left(\sum_{k=1}^{n} A\left(\varepsilon_{i k} d_{k}\right)\right)\right\|_{Y}^{q}\right)^{p^{*}} d P(\omega)\right)^{1 / p^{*}} \\
& \leq\left(\int_{\Omega}\left(\sum_{i=1}^{\infty} 2^{-i q}\left\|\sum_{k=1}^{n} A\left(\varepsilon_{i k} d_{k}\right)\right\|_{Y}^{q}\right)^{p^{*}} d P(\omega)\right)^{1 / p^{*}} \\
& \leq \sum_{i=1}^{\infty}\left(\int_{\Omega} 2^{-i q p^{*}}\left\|\sum_{k=1}^{n} A\left(\varepsilon_{i k} d_{k}\right)\right\|_{Y}^{q p^{*}} d P(\omega)\right)^{1 / p^{*}} \\
& =\sum_{i=1}^{\infty} 2^{-i q}\left(\int_{\Omega}\left\|\sum_{k=1}^{n} A\left(\varepsilon_{i k} d_{k}\right)\right\|_{Y}^{q p^{*}} d P(\omega)\right)^{1 / p^{*}} \\
& \leq \sum_{i=1}^{\infty} 2^{-i q} C^{q}\left(\int_{\Omega}\left\|\sum_{k=1}^{n} d_{k}\right\|_{X} d P(\omega)\right)^{1 / p^{*}}
\end{aligned}
$$

Thus

$$
\left\|A g_{n}\right\|_{L_{p}(Y)} \leq C\left(2^{q}-1\right)^{-1 / q}\left\|f_{n}\right\|_{L_{p}(X)},
$$

where $C=C(p)$ is the UMD constant of $A$. This completes the proof.

We remark that Burkholder [3] was able to show that the MT constant of a Banach space $X$ equals the UMD constant of $X$. We have been unable to get a result of that strength.

3. The $\varsigma$-characterization of an MT operator. Burkholder [3] showed that a Banach space $X$ is an MT space if and only if there exists a symmetric, biconvex function $\varsigma: X \times X \rightarrow \mathbf{R}$ such that $\varsigma(0,0)>0$ and $\varsigma(x+y) \leq\|x+y\|$ if $\|y\| \geq 1$. We now prove that a similar result holds for an MT operator $A: X \rightarrow Y$. By Theorem 1 we may assume $A$ is an UMD operator. We assume $A$ is nonzero to avoid technical complications in our proof.

THEOREM 2. Let $A: X \rightarrow Y$ be a nonzero continuous linear operator from a Banach space $X$ into a quasi-Banach space $Y$ with q-subadditive quasi-norm, $0<q \leq 1$. Then $A$ is an UMD operator if and only if there exists a symmetric, biconvex function $\varsigma: X \times X \rightarrow \mathbf{R}$ ( $\varsigma$ depending on $A$ as defined below) so that $\varsigma(0,0)>0$ and

$$
\varsigma(x, z) \leq\|x+z\|_{X} \quad \text { if }\|A(z)\|_{Y} \geq 1 \text {. }
$$


Proof. Let $x, z \in X$ and define $M(x, z)$ to be the class of all martingales $f$ with $f_{1}=x$ so that for some $\varepsilon=\left(1, \varepsilon_{2}, \varepsilon_{3}, \ldots\right)$ the transform $g$ of $f$ by $\varepsilon$ satisfies

$$
P\left(\left\|A\left(g_{n}\right)-A(z)\right\|_{Y} \geq 1 \text { for some } n \geq 1\right)=1 \text {. }
$$

Define

$$
\psi(x, z)=\inf \left\{\|f\|_{L_{1}(X)}: f \in M(x, z)\right\} .
$$

Using arguments similar to those in [3, pp. 1003-1004] one can show that $\psi$ has these properties:

(1) $\psi(x, z)=\psi(x, 2 x-z)$,

(2) $\psi(\cdot, z)$ is convex,

(3) $\psi(x, z) \leq\|x\|_{X}$ if $\|A(z)\|_{Y} \geq 1$. from

Now define $\zeta$ by $\zeta(x, z)=2 \psi((x+z) / 2, z)$. The fact that $\zeta$ is symmetric follows

$$
\begin{aligned}
\varsigma(x, z) & =2 \psi((x+z) / 2, z) \\
& =2 \psi((x+z) / 2, x+z-z)=\varsigma(z, x) .
\end{aligned}
$$

That $\zeta$ is biconvex follows from (2) and the symmetry of $\zeta$. If $\|A(z)\|_{Y} \geq 1$, then by (3) we have

$$
\varsigma(x, z)=2 \psi\left(\frac{x+z}{2}, z\right) \leq 2\left\|\frac{x+z}{2}\right\|_{X}=\|x+z\|_{X} .
$$

Now suppose $A$ is an UMD operator. Let $f \in M(0,0)$ with corresponding transform $g$. Clearly then $(A g)^{*} \geq 1$ a.e. By Theorem 1.1 of $[\mathbf{1}]$ there is a constant $C>0$ so that $\|f\|_{L_{1}(X)} \geq C$. Thus $\psi(0,0)=\varsigma(0,0)>0$.

Assume $\psi(0,0)>0$. Let $f$ be a martingale, let $\varepsilon$ be a sequence of signs and $g$ the transform of $f$ by $\varepsilon$. As in Remark 1.1 of [3], we may assume $f_{1}=0$. Suppose $(A g)^{*}>1$ a.e. Then by the definition of $\psi$, we have $\|f\|_{L_{1}(X)} \geq \psi(0,0)>0$. By Theorem 1.1 of $[\mathbf{1}]$, this implies that $A$ is an MT (thus also UMD) operator. This completes the proof.

We remark that out $\zeta$ depends on $A$. Burkholder $[3]$ shows that $X$ is an MT space if and only if any $\zeta$ which is symmetric, biconvex and for which $\zeta(x, z) \leq\|x+z\|_{X}$ for $\|z\|_{X} \geq 1$ satisfies $\zeta(0,0)>0$. In our setting given any $\zeta$ as in Theorem 2 we must show the following: If $\|A(x-z)\|_{Y} \geq 1$, then

$$
\max \left\{\|A z\|_{Y},\|A(2 x-z)\|_{Y}\right\} \geq 1 \text {. }
$$

But, for $0<q<1$, the most we can conclude is

$$
\max \left\{\|A z\|_{Y},\|A(2 x-z)\|_{Y}\right\} \geq 2^{1-1 / q} .
$$

Since $2^{1-1 / q}<1$, we cannot invoke the properties of this $\zeta$ to show that $A$ is an UMD operator (compare to [3, p. 1005]).

\section{REFERENCES}

1. J. Bourgain and W. J. Davis, Martingale transforms and complex uniform convexity, preprint.

2. D. L. Burkholder, A sharp inequality for martingale transforms, Ann. Probab. 7 (1979), 858-863.

3. __ A geometrical characterization of Banach spaces in which martingale difference sequences are unconditional, Ann. Probab. 9 (1981), 997-1011.

4. S. Rolewicz, Metric linear spaces, Monografie Matematczyne, Tom 56, PWN, Warsaw, 1972. 